\title{
Measurement of Mixed Venous Oxygen Tension in Premature Infants by a Rebreathing Method
}

\author{
A. N. Krauss ${ }^{[16]}$, And P. A. M. Auld \\ Department of Pediatrics, Cornell University Medical College, New York, New York, USA
}

\begin{abstract}
Extract
The $\mathrm{CO}_{2}$ and oxygen tensions of mixed venous blood were determined by the rebreathing method of Cerretelli et al. in a group of 21 low birth weight infants. Venous $\mathrm{P}_{\mathrm{O}_{2}}$ ranged from 10 to $43 \mathrm{~mm} \mathrm{Hg}$, with a mean of $28 \mathrm{~mm} \mathrm{Hg}$, and showed no tendency to change with size or age. Cardiac output was calculated from these data according to the Fick principle. Values of $50-100 \mathrm{ml} / \mathrm{min} / \mathrm{kg}$ were obtained in newborn low birth weight infants; these rise with age to $300-500 \mathrm{ml} / \mathrm{min} / \mathrm{kg}$, at which time they are comparable with term infants.
\end{abstract}

\section{Speculation}

The method described herein is practical, simple, and accurate. It provides further quantitative data to characterize healthy and distressed newborns with regard to cardiac output and $\dot{\mathrm{V} A} / \dot{\mathrm{Q}}$ relations.

\section{Introduction}

The recent development of bloodless techniques for measurement of mixed venous oxygen tension is an important development. In this method, the lung is used as an aerotonometer to equilibrate alveolar gas and mixed venous blood. The oxygen and carbon dioxide tensions of this gas are continuously sampled. When no further transfer of gas between blood and gas phases occurs, the gas in the rebreathing bag is in equilibrium with the mixed venous blood. The identity between mixed venous oxygen and alveolar gas tensions during rebreathing has been verified by Cerretelli $e t$ al. [3] and Denison et al. [4]. The study reported herein used the method of Cerretelli and co-workers [2] as modified by Farhi and co-workers [8].

\section{Materials and Methods}

The subjects were 21 newborn infants ranging in age from $4 \mathrm{hr}$ to 60 days and with birth weights from $1050 \mathrm{~g}$ to $3600 \mathrm{~g}$. Infants 13 and 15 were small for dates; all the others were the appropriate weight for the gestational age. All were healthy at the time of study. Infants 11 and 14 received transfusions because of anemia. The tests were carried out with the infants swaddled in blankets to maintain body temperature. First, arterial blood was obtained from the temporal or right radial artery with the infant at rest, and $\mathrm{pH}, \mathrm{P}_{\mathrm{CO}_{2}}$, and $\mathrm{P}_{\mathrm{O}_{2}}$ were determined [12]. The values obtained were corrected to the infant's body temperature. Immediately following, endtidal $\mathrm{CO}_{2}\left(\mathrm{PA}_{\mathrm{CO}_{2}}\right)$ was determined with a rapid response infrared $\mathrm{CO}_{2}$ meter [13].

To obtain mixed venous $\mathrm{P}_{\mathrm{CO}_{2}}\left(\mathrm{P}_{\mathrm{V} \mathrm{O}_{2}}\right)$ and $\mathrm{P}_{\mathrm{O}_{2}}\left(\mathrm{P}_{\mathrm{v}_{2}}\right)$, the infant rebreathed from a bag containing $75-125 \mathrm{ml}$ of a gas mixture of $8 \% \mathrm{CO}_{2}$ in nitrogen. During rebreathing, gas was sampled from the bag at a rate of $100 \mathrm{ml} / \mathrm{min}$, first through a microcuvette containing a Clark oxygen electrode [12] and then through an infrared $\mathrm{CO}_{2}$ analyzer. The two systems were in series and the signals from each were recorded on a Sanborn 
recorder. The oxygen electrode was covered with a 3- $\mu$ Teflon membrane to increase the response time (90\% response time less than $1 \mathrm{sec}$ ). This membrane was stable for at least $30 \mathrm{~min}$ at $37^{\circ}$. The linearity of the system was established.

During the test, equilibrium between pulmonary venous blood and alveolar gas was achieved when a plateau was recorded for $\mathrm{O}_{2}$ and $\mathrm{CO}_{2}$ (Fig. 1). The values presented for $P \bar{v}_{\mathrm{CO}_{2}}$ and $P \overline{\mathrm{v}}_{\mathrm{O}_{2}}$ are the average of three runs which varied by less than $0.5 \%$. A 5 -min rest period was allowed between each run, during which time the calibration of the oxygen electrode was rechecked. The entire procedure required less than 30 min and no ill effects were notcd. If no platcau for either $\mathrm{P}_{\mathrm{O}_{2}}$ or $\mathrm{P}_{\mathrm{CO}_{2}}$ was obtained within $10 \mathrm{scc}$, the run was rejected. This period of time was believed to be well within the recirculation time for these subjects.

\section{Evaluation of the Method}

The theoretical and practical limitations of measuring $\mathrm{P}_{\mathrm{V}_{2}}$ and $\mathrm{P}_{\mathrm{V}_{\mathrm{CO}}}$ have been discussed by Cerretelli et al. [2] and Farhi and Haab [7]. A large a- $\mathrm{ACO}_{2}$ gradient with concurrent large dead space/tidal volume ratios may result in a delayed or elevated plateau for $\mathrm{CO}_{2}$. This is not a problem with oxygen because of the relative slopes of the dissociation curves for $\mathrm{CO}_{2}$ and oxygen. Recirculation may occur before a plateau is reached. This occurs in situations in which cardiac output is elevated or in which respiratory rate during rebreathing is slow. If a diffusion barrier exists, the achievement of an oxygen plateau may be delayed. This is important for oxygen because of the low tensions involved.

Cerretelli et al. [3] and Denison et al. [4] have demonstrated by catheterization studies an identity between blood and gas oxygen tensions when an equilibrium plateau is reached during rebreathing. Gurtner et al. [9] and Denison et al. [4] have shown that this method overestimates mixed venous $\mathrm{CO}_{2}\left(\mathrm{P}_{\mathrm{V}_{\mathrm{CO}}}\right)$ by about $5 \mathrm{~mm}$ $\mathrm{Hg}$. This results in only a slight underestimation of mixed venous oxygen saturation $\left(\mathrm{S}_{\mathrm{O}_{2}}\right)$ and oxygen content $\left(\mathrm{C}_{\mathrm{V}_{2}}\right)$ when Dill's nomogram is used.

The 3- $\mu$ Teflon membrane used to improve the response time of the oxygen electrode is delicate and subject to both tearing and drying. Care is needed in handling it. A $90 \%$ response time of $0.3-0.5 \mathrm{sec}$ is obtained with a $3-\mu$ Teflon membrane, however.

\section{Results}

Data describing the subjects studied, measured values of mixed venous $\mathrm{O}_{2}$ and $\mathrm{CO}_{2}$, and arterial $\mathrm{P}_{\mathrm{O}_{2}}$ and $\mathrm{P}_{\mathrm{CO}_{2}}$ appear in Table I. Mixed venous $\mathrm{P}_{\mathrm{O}_{2}}$ varied from 10 to $43 \mathrm{~mm} \mathrm{Hg}$ and showed no relation to postnatal age, body size, or arterial oxygen tension. Data derived from the measured values appear in Table II.

\section{Discussion}

The importance of knowledge of the mixed venous $\mathrm{P}_{\mathrm{O}_{2}}$ is that cardiac output and venous admixture may then be calculated according to the Fick principle. These calculations may be made from the data in Table I if it is assumed that: (1) a steady state exists during the period of sampling, and (2) the oxygen consumption of these infants is similar to others studied in our laboratory. Derivation of the cardiac output and venous admixture from the data could also be affected by the fact that arterial and venous gas tensions wcre determined sequentially rather than simultancously, even though great care was taken to assure a steady state. The assumption of oxygen consumption can introduce an crror, but the values used were obtained by us on a similar group of babies [10] under similar conditions and they closely agree with the values of others.

Previous workers have had to assume mixed venous oxygen tension in order to calculate cardiac output and venous admixture. This is more likely to cause a large error than the factors already discusscd because of the steep slope of the hemoglobin-oxygen dissociation curvc at venous tensions. Small changes in oxygen tension result in large changes of saturation. Blood at arterial $\mathrm{P}_{\mathrm{O}_{2}}$ tensions is less susceptible to this effect.

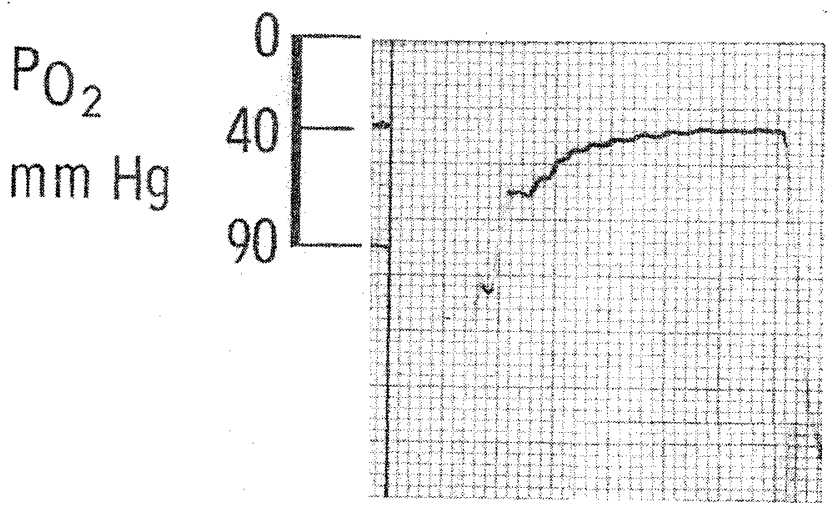

\section{Seconds}

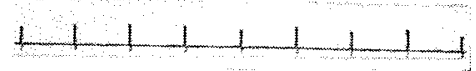

Fig. 1. Alveolar $\mathrm{P}_{\mathrm{O}_{2}}$ tension obtained during rebreathing. 
Table I. Mixed venous oxygen and carbon dioxide data ${ }^{1}$

\begin{tabular}{|c|c|c|c|c|c|c|c|c|c|c|c|c|c|c|}
\hline $\begin{array}{l}\text { Case } \\
\text { no. }\end{array}$ & Birth wt, g & Study wt, $\mathrm{g}$ & $\begin{array}{l}\text { Study } \\
\text { age, } \\
\text { days }\end{array}$ & $\begin{array}{c}\text { Length, } \\
\mathrm{cm}\end{array}$ & $\begin{array}{l}\text { Body } \\
\text { surface } \\
\text { area, } m^{2}\end{array}$ & $\begin{array}{c}\text { Hemato- } \\
\text { crit, } \%\end{array}$ & $\begin{array}{c}\mathrm{O}_{2} \\
\text { capacity, } \\
\text { vol } / 100 \mathrm{ml}\end{array}$ & $\begin{array}{l}\mathrm{PaO}_{2} \\
\mathrm{~mm} \mathrm{Hg}\end{array}$ & $\begin{array}{l}\mathrm{PaCO}_{2}, \\
\mathrm{~mm} \mathrm{Hg}\end{array}$ & $\begin{array}{c}\mathrm{PvO}_{2} \\
\mathrm{~mm} \mathrm{Hg}\end{array}$ & $\begin{array}{l}\mathrm{P} \mathrm{vCO}_{2}, \\
\mathrm{~mm} \mathrm{Hg}\end{array}$ & $\begin{array}{c}\mathrm{CaO}_{2} \\
\mathrm{vol} / 100 \mathrm{ml}\end{array}$ & $\begin{array}{c}\mathrm{C} \mathrm{CuO}_{2} \\
\mathrm{vol} / 100 \\
\mathrm{ml}\end{array}$ & $\begin{array}{c}\mathrm{a}-\mathrm{vo}_{2}, \\
\mathrm{vol} / 100 \\
\mathrm{ml}\end{array}$ \\
\hline \multirow[t]{5}{*}{1} & 1300 & 1300 & 1 & 39 & 0.118 & 52 & 23.9 & 70.0 & 42.0 & 30 & 43 & 22.5 & 15.0 & 7.5 \\
\hline & & 1240 & 2 & 39 & 0.118 & 44 & 20.3 & 79.1 & 27.3 & 27 & 41 & 19.5 & 11.5 & 8.0 \\
\hline & & 1520 & 21 & 42 & 0.141 & 27 & 12.4 & 84.5 & 31.5 & 27 & 38 & 12.0 & 7.8 & 4.2 \\
\hline & & 1520 & 22 & 42 & 0.141 & 28 & 12.9 & 86.5 & 34.5 & 24 & 46 & 12.3 & 5.7 & 6.6 \\
\hline & & 1900 & 34 & 44 & 0.157 & 25 & 10.5 & 83.6 & 34.9 & 18 & 44 & 10.2 & 3.7 & 6.5 \\
\hline 2 & 2480 & 3460 & 28 & & & 29 & 13.4 & 76.9 & 32.0 & 37 & 43 & 12.5 & 10.6 & 1.9 \\
\hline 3 & 1900 & 2470 & 30 & 43 & 0.152 & 33 & 15.2 & 89.0 & 29.0 & 31 & 44 & 14.5 & 9.8 & 4.7 \\
\hline 4 & 2600 & 2400 & 8 & 40 & 0.126 & 32 & 14.7 & 84.9 & 30.0 & 33 & 45 & 14.0 & 8.8 & 5.2 \\
\hline \multirow[t]{2}{*}{5} & 1616 & 1510 & 1.5 & 41 & 0.135 & 36 & 16.6 & 57.1 & 35.2 & 27 & 50 & 15.0 & 8.7 & 6.3 \\
\hline & & 1580 & 8 & 41 & 0.135 & 43 & 19.8 & 50.6 & 38.8 & 24 & 52 & 17.0 & 9.0 & 8.0 \\
\hline \multirow[t]{3}{*}{6} & 1440 & 1440 & $6 \mathrm{hr}$ & 40 & 0.126 & 45 & 20.7 & 48.1 & 32.8 & 16 & 49 & 17.5 & 5.5 & 12.0 \\
\hline & & 1350 & 6 & 40 & 0.126 & 45 & 20.7 & 82.0 & 32.7 & 21 & 53 & 19.7 & 8.0 & 11.7 \\
\hline & & 1490 & 13 & 40 & 0.126 & 40 & 18.4 & 55.5 & 37.8 & 20 & 46 & 16.5 & 7.0 & 9.5 \\
\hline \multirow[t]{2}{*}{7} & 2200 & 2200 & $4 \mathrm{hr}$ & 45 & 0.166 & 51 & 23.7 & 62.0 & 37.0 & 26 & 44 & 21.8 & 11.8 & 10.0 \\
\hline & & 2140 & 1 & 45 & 0.166 & 43 & 19.8 & 74.6 & 31.5 & 27 & 47 & 18.5 & 10.8 & 7.7 \\
\hline \multirow[t]{2}{*}{8} & 1060 & 1070 & 35 & & & 31 & 14.8 & 63.0 & 36.0 & 32 & 45 & 13.5 & 9.7 & 3.8 \\
\hline & & 1320 & 60 & $4 \mathrm{I}$ & 0.135 & 20 & 9.2 & 78.0 & 33.0 & 33 & 39 & 8.8 & 6.4 & 2.4 \\
\hline 9 & 1160 & 1520 & 34 & & & 26 & 12.0 & 71.0 & 41.0 & 29 & 45 & 11.1 & 7.2 & 3.9 \\
\hline \multirow[t]{4}{*}{10} & 1050 & 970 & 8 & 38 & 0.113 & 42 & 19.4 & 56.6 & 40.4 & 29 & 42 & 17.0 & 12.0 & 5.0 \\
\hline & & 1250 & 29 & 40 & 0.126 & 27 & 12.4 & 93.0 & 34.0 & 30 & 41 & 11.9 & 7.9 & 4.0 \\
\hline & & 1440 & 40 & 43 & 0.149 & 23 & 10.6 & 78.8 & 30.6 & 31 & 47 & 10.0 & 6.5 & 3.5 \\
\hline & & 1690 & 47 & 43 & 0.149 & 20 & 9.2 & 80.0 & 32.0 & 26 & 45 & 8.8 & 4.9 & 3.9 \\
\hline \multirow[t]{3}{*}{11} & 1390 & 1530 & 34 & & & 19 & 8.8 & 63.0 & 36.0 & 19 & 46 & 8.0 & 3.1 & 4.9 \\
\hline & & 1780 & 40 & & & 18 & 8.3 & 69.5 & 31.1 & 18 & 42 & 7.8 & 3.0 & 4.8 \\
\hline & & 1810 & 43 & & & 24 & 11.1 & 67.6 & 33.0 & 37 & 34 & 10.4 & 7.9 & 2.5 \\
\hline \multirow[t]{2}{*}{12} & 1440 & 1690 & 29 & & & 21 & 9.7 & 68.6 & 40.0 & 29 & 44 & 8.9 & 5.9 & 3.0 \\
\hline & & 2160 & 47 & 44 & 0.157 & 21 & 9.7 & 52.0 & 36.0 & 28 & 55 & 8.5 & 5.1 & 3.4 \\
\hline \multirow[t]{2}{*}{13} & 1560 & 1560 & 1 & 38 & 0.112 & 63 & 29.0 & 84.0 & 28.5 & 24 & 43 & 28.0 & 14.0 & 14.0 \\
\hline & & 1880 & 21 & 42 & 0.141 & 40 & 18.4 & 85.3 & 31.2 & 30 & 42 & 17.7 & 11.5 & 6.2 \\
\hline \multirow[t]{2}{*}{14} & 1660 & 1900 & 24 & & & 21 & 9.7 & 53.0 & 37.0 & 41 & 43 & 8.5 & 7.5 & 1.0 \\
\hline & & 1960 & 27 & & & 32 & 14.7 & 57.0 & 32.0 & 41 & 44 & 13.3 & 11.3 & 2.0 \\
\hline \multirow[t]{2}{*}{15} & 1800 & 1800 & 2 & 43 & 0.149 & 55 & 25.3 & 77.1 & 34.4 & 34 & 42 & 23.5 & 17.7 & 5.8 \\
\hline & & 2130 & 12 & 45 & 0.166 & 39 & 17.9 & 79.0 & 32.0 & 10 & 49 & 17.0 & 10.2 & 6.8 \\
\hline 16 & 2880 & 2880 & 2 & 49 & 0.200 & 55 & 25.3 & 69.9 & 32.8 & 18 & 36 & 24.0 & 9.7 & 14.3 \\
\hline \multirow[t]{3}{*}{17} & 1790 & 1680 & 3 & 42 & 0.141 & 46 & 21.2 & 79.0 & 36.6 & 22 & $5 \mathrm{I}$ & 20.5 & 8.8 & 11.7 \\
\hline & & 1680 & 4 & 42 & 0.141 & 44 & 20.2 & 100.8 & 26.3 & 40 & 37 & 20.0 & 15.7 & 4.3 \\
\hline & & 1680 & 10 & 42 & 0.141 & 45 & 20.7 & 65.0 & 35.4 & 28 & 44 & 19.5 & 12.0 & 7.5 \\
\hline \multirow[t]{3}{*}{18} & 1700 & 1640 & 3 & 43 & 0.149 & 58 & 26.7 & 77.0 & 33.6 & 41 & 40 & 25.0 & 8.2 & 16.8 \\
\hline & & 1640 & 4 & 43 & 0.149 & 56 & 25.8 & 65.5 & 36.0 & 26 & 38 & 23.5 & 14.5 & 9.0 \\
\hline & & 1590 & 10 & 43 & 0.149 & 57 & 26.2 & 76.9 & 34.9 & 27 & 42 & 24.5 & 14.7 & 9.8 \\
\hline \multirow[t]{2}{*}{19} & 1330 & 1310 & 24 & 38 & 0.112 & 32 & 14.7 & 53.0 & 37.6 & 27 & 64 & 13.0 & 7.1 & 5.9 \\
\hline & & 1560 & 34 & 39 & 0.118 & 27 & 12.4 & 72.0 & 37.5 & 26 & 44 & 11.6 & 6.8 & 4.8 \\
\hline 20 & 3600 & 3560 & 1 & 46 & 0.174 & 54 & 25.0 & 80.9 & 30.5 & 34 & 42 & 24.0 & 17.5 & 6.5 \\
\hline 21 & 2540 & 2300 & 6 & 44 & 0.157 & 35 & 16.2 & 69.6 & 29.2 & 43 & 45 & 15.2 & 12.5 & 2.7 \\
\hline
\end{tabular}

$1 \mathrm{PaO}_{2}$ : Arterial oxygen tension. $\mathrm{PaCO}_{2}$ : Arterial carbon dioxide tension. $\mathrm{P}_{\mathrm{v}_{2}}$ : Mixed venous oxygen tension. $\mathrm{P} \overline{\mathrm{v}}_{\mathrm{CO}_{2}}: \mathrm{Mixed}$ venous carbon dioxide tension. $\mathrm{Ca}_{2}$ : Arterial oxygen content. $\mathrm{C}_{\mathrm{v}_{2}}$ : Venous oxygen content. $\mathrm{a}-\mathrm{v}_{\mathrm{O}_{2}}$ : Arteriovenous oxygen difference.

With these limitations, cardiac output may be calculated according to the Fick equation:

$$
\dot{\mathrm{Q}}_{\mathrm{s}}=\frac{\dot{\mathrm{V}}_{\mathrm{O}_{2}}}{\mathrm{Ca}_{\mathrm{O}_{2}}-\mathrm{C}_{\mathrm{O}_{2}}} \times 100
$$

where $\dot{\mathrm{V}}_{\mathrm{O}_{2}}=$ oxygen consumption, milliliters per minute (standard temperature and pressure, dry); $\mathrm{Ca}_{\mathrm{O}_{2}}=$ pre- ductal arterial $\mathrm{O}_{2}$ content; $\mathrm{C}_{\mathrm{O}}{ }_{\mathrm{O} 2}=$ mixed venous oxygen content; and $\dot{Q} s=$ systemic flow, milliliters per minute. Oxygen content was derived from Dill et al.'s nomogram [5] as modified for fetal blood by Edwards and Ross [6]. Oxygen capacity was determined by using Peters' factor [11], hematocrit $\times 0.46$. Pulse rate taken at the end of the experiment was used to calculate stroke vol- 
Table II. Calculation of cardiac output and venous admixture ${ }^{1}$

\begin{tabular}{|c|c|c|c|c|c|c|c|c|}
\hline $\begin{array}{c}\text { Case } \\
\text { no. }\end{array}$ & $\begin{array}{c}\dot{\mathrm{Q}}_{\mathrm{s}} \\
\mathrm{ml} / \mathrm{min}\end{array}$ & $\begin{array}{c}\dot{\mathrm{Qs}}, \\
\mathrm{ml} \\
\mathrm{min} / \mathrm{kg}\end{array}$ & $\underset{\mathrm{min} / \mathrm{m}^{2}}{\dot{\mathrm{Q}}}$ & $\begin{array}{c}\dot{\mathrm{V}}_{2} \\
{[10]} \\
\text { (as- } \\
\text { sumed), } \\
\text { ml/min, } \\
\text { STPD2 }^{2}\end{array}$ & $\begin{array}{c}\text { Venous } \\
\text { admix- } \\
\text { ture, } \\
\%\end{array}$ & $\begin{array}{c}\text { Pulse } \\
\text { beats/ } \\
\text { min }\end{array}$ & $\begin{array}{c}\mathrm{SaO}_{2} \\
\%\end{array}$ & $\begin{array}{c}\mathrm{Sv}_{\mathrm{V}_{2}} \\
\%\end{array}$ \\
\hline \multirow[t]{5}{*}{1} & 116 & 81 & 985 & 8.7 & 16 & 148 & 94 & 63 \\
\hline & 109 & 88 & 925 & 8.7 & 9 & & 96 & 58 \\
\hline & 291 & 191 & 2050 & 12.2 & 9 & 160 & 97 & 63 \\
\hline & 185 & 122 & 1310 & 12.2 & 8 & & 95 & 44 \\
\hline & 234 & 123 & 1440 & 15.2 & 4 & 156 & 97 & 35 \\
\hline 2 & 1600 & 440 & & 30.4 & 32 & & 93 & 79 \\
\hline 3 & 462 & 187 & 3039 & 21.7 & 13 & & 95 & 64 \\
\hline 4 & 405 & 169 & 3214 & 21.1 & 14 & & 95 & 66 \\
\hline \multirow[t]{2}{*}{5} & 161 & 107 & 1190 & 10.1 & 20 & 140 & 90 & 58 \\
\hline & 149 & 95 & 1200 & 11.9 & 25 & & 86 & 45 \\
\hline \multirow[t]{3}{*}{6} & 79 & 54 & 630 & 9.4 & $2 \mathrm{I}$ & 136 & 85 & 32 \\
\hline & 85 & 63 & 675 & 9.9 & 11 & & 95 & 39 \\
\hline & 116 & 78 & 920 & 11.0 & 17 & 136 & 90 & 38 \\
\hline \multirow[t]{2}{*}{7} & 147 & 67 & 885 & 14.7 & 16 & 160 & 92 & 50 \\
\hline & 214 & 97 & 1290 & 16.5 & 14 & 140 & 93 & 55 \\
\hline \multirow[t]{2}{*}{8} & 214 & 199 & & 8.1 & 26 & 148 & 91 & 66 \\
\hline & 443 & 336 & 3280 & 10.6 & 14 & & 96 & 70 \\
\hline 9 & 298 & 196 & & 11.6 & 19 & & 93 & 60 \\
\hline \multirow[t]{4}{*}{10} & 140 & 143 & 1240 & 7.0 & 32 & 145 & 88 & 62 \\
\hline & 250 & 200 & 1980 & 10.0 & 11 & & 96 & 64 \\
\hline & 311 & 216 & 2040 & 10.9 & 15 & 150 & 94 & 61 \\
\hline & 328 & 194 & 2201 & 12.8 & 8 & 150 & 96 & 56 \\
\hline \multirow[t]{3}{*}{11} & 250 & 164 & & 12.2 & 14 & 158 & 91 & 35 \\
\hline & 310 & 174 & & 14.9 & 9 & & 94 & 36 \\
\hline & 580 & 321 & & 14.5 & 18 & & 94 & 71 \\
\hline \multirow[t]{2}{*}{12} & 450 & 267 & & 13.5 & 21 & 130 & 92 & 61 \\
\hline & 509 & 236 & 3240 & 17.3 & 26 & & 88 & 53 \\
\hline \multirow[t]{2}{*}{13} & 80 & 53 & 705 & 11.2 & 7 & 138 & 97 & 48 \\
\hline & 266 & 141 & 1900 & 16.5 & 10 & 170 & 96 & 63 \\
\hline \multirow[t]{2}{*}{14} & 2170 & 1125 & & 21.7 & 55 & 192 & 88 & 77 \\
\hline & 1085 & 554 & & 21.7 & 41 & & 90 & 77 \\
\hline \multirow[t]{2}{*}{15} & 225 & 125 & 1510 & 13.0 & 24 & 130 & 93 & 70 \\
\hline & 266 & 125 & 1600 & 18.1 & 12 & 150 & 95 & 57 \\
\hline 16 & 151 & 52 & 755 & 21.6 & 8 & 150 & 95 & 38 \\
\hline \multirow[t]{3}{*}{17} & 108 & 64 & 754 & 12.6 & 6 & & 97 & 42 \\
\hline & 293 & 174 & 2080 & 12.6 & 4 & 128 & 99 & 78 \\
\hline & 175 & 104 & 1240 & 13.1 & 14 & 144 & 94 & 58 \\
\hline \multirow[t]{3}{*}{18} & 73 & 45 & 490 & 12.3 & 9 & & 94 & 31 \\
\hline & 137 & 84 & 920 & 12.3 & 20 & 144 & 91 & 56 \\
\hline & 127 & 80 & 850 & 12.4 & 15 & 156 & 94 & 56 \\
\hline \multirow[t]{2}{*}{19} & 178 & 136 & 1590 & 10.5 & 22 & & 88 & 48 \\
\hline & 260 & 167 & 2200 & 12.5 & 14 & 172 & 94 & 55 \\
\hline 20 & 368 & 103 & 2115 & 23.9 & 13 & 150 & 96 & 70 \\
\hline 21 & 663 & 288 & 4220 & 17.9 & 27 & 144 & 94 & 77 \\
\hline
\end{tabular}

1 S: Systemic flow. $\dot{\mathrm{V}}_{\mathrm{O}_{2}}$ : Oxygen consumption. $\mathrm{Sa}_{2}$ : Arterial oxygen saturation. $\mathrm{S}_{\mathrm{O}_{2}}$ : Venous oxygen saturation.

${ }^{2}$ STPD : Standard temperature and pressure, dry.

ume. Venous admixture was calculated using the Berggren shunt equation [1]:

$$
\dot{\mathrm{Q}} \mathrm{s} / \dot{\mathrm{Q}} \mathrm{t}=\left(\mathrm{Cc}_{\mathrm{O}_{2}}-\mathrm{Ca}_{\mathrm{O}_{2}}\right) /\left(\mathrm{Ci}_{\mathrm{O}_{2}}-\mathrm{C}_{\mathrm{V}_{2}}\right)
$$

The results of these calculations are seen in Table II. Systemic cardiac output was lowest during the 1st week of life, ranging from $50-100 \mathrm{ml} / \mathrm{min} / \mathrm{kg}$ or $500-1500$ $\mathrm{ml} / \mathrm{min} / \mathrm{m}^{2}$. An increase in calculated systemic output was noted as the infants became older. Venous admixture was under $30 \%$ in most cases, but showed no tendency to rise or fall with time. The cardiac output calculations are recorded as systemic blood flow because the presence of a right-to-left shunt through the foramen ovale is a possibility, particularly in the first days of life. A right-to-left ductus shunt would appear to be excluded because of a sampling site (right radial or temporal artery) proximal to its origin.

\section{References and Notes}

1. BERgGren, S. M.: $\mathrm{O}_{2}$ deficit of arterial blood caused by nonventilatory parts of the lung. Acta Physiol. Scand. (Suppl. I1), 4 : 1 (1942).

2. Cerretelli, P., Cruz, J. C., Farhi, L. E., a.do Rahn, H.: Determinations of mixed venous $\mathrm{O}_{2}$ and $\mathrm{CO}_{2}$ tensions and cardiac output by a rebreathing method. Resp. Physiol., 1: 258 (1966).

3. Cerretelli, P., DiPrampero, P. E., and Rennie, D. W.: Measurement of mixed venous oxygen tension by a modified rebreathing method. J. Appl. Physiol., 28: 707 (1970).

4. Denison, D., Edwards, R. H. T., Jones, G., and Pope, H.: Direct and rebreathing estimates of the $\mathrm{O}_{2}$ and $\mathrm{CO}_{2}$ pressures in mixed venous blood. Resp. Physiol., 7: 326 (1969).

5. Dill, D. B., Edwards, H. T., and Consolazio, W. V.: Blood as a physicochemical system. XI. Man at rest. J. Biol. Chem., 118: 635 (1937).

6. Edwards, M. J., and Ross, B.: Graphical representation of respiratory gas dissociation characteristics of fetal and maternal blood. J. Appl. Physiol., 14: 454 (1959).

7. FARHi, L. E., AND HAAB, P.: Mixed venous blood gas tensions and cardiac output by "bloodless" methods: recent developments and appraisal. Resp. Physiol., 2: 225 (1967).

8. Farhi, L. E., Chinet, A., and HaAb, P.: Pression partielle d'oxygene du sang veineux mele chez l'homme, en plaine et en haute altitude. J. Physiol. (Paris), 58: 516 (1966).

9. Gurtner, G. H., Song, S. H., and Farhi, L. E.: Alveolar and mixed venous $\mathrm{P}_{\mathrm{CO}_{2}}$ difference under conditions of no gas exchange. Resp. Physiol., 7: 173 (1969).

10. Krauss, A. N., ANd Auld, P. A. M.: Metabolic requirements of low birth weight infants. J. Pediat., 75: 952 (1969).

11. Peters, J. P., Brelgar, H. A., and Eisenman, A. J.: Studies of the $\mathrm{CO}_{2}$ absorption curve of human blood. IV. The relation of the hemoglobin content of blood to the form of the $\mathrm{CO}_{2}$ absorption curve. J. Biol. Chem., 58: 749 (1924).

12. Instrumentation Laboratory Ultramicro system, Instrumentation Laboratory, Inc., Lexington, Mass.

13. Beckman Instruments, Inc., Palo Alto, Calif.

14. Dr. P. A. M. Auld holds an Investigatorship of the Health Research Council of the City of New York, under Contract no. I-236.

15. Supported by Public Health Service Research Grants nos. HD-02644 and HE-09410.

16. Requests for reprints should be addressed to: A. N. Krauss, M.D., Department of Pediatrics, Cornell University Medical College, 525 East 68th Street, New York, N. Y. 10021 (USA).

17. Accepted for publication July 9, 1971. 